\title{
Exploiting long-ranged order in quasiperiodic structures for broadband plasmonic excitation
}

\author{
B. le Feber, ${ }^{\text {a) }}$ J. Cesario, H. Zeijlemaker, N. Rotenberg, and L. Kuipers \\ Center for Nanophotonics, FOM Institute for Atomic and Molecular Physics (AMOLF), Science Park 104, \\ 1098 XG Amsterdam, The Netherlands
}

(Received 28 February 2011; accepted 28 April 2011; published online 19 May 2011)

\begin{abstract}
A comparison of transmission spectra from periodic, quasiperiodic, and randomly spaced slit arrays in thick gold films reveals resonant plasmonic excitations that arise solely due to the long-range order of the quasiperiodic structures. Specifically, first-order plasmonic resonances at the air-gold interface of the quasiperiodic arrays are identified at a broader range of wavelengths than those observed from periodic structures with the same average slit distance. Thus, a quasiperiodic plasmonic coupler that couples both visible and near-infrared light to surface plasmon polaritons is designed and demonstrated. (C) 2011 American Institute of Physics. [doi:10.1063/1.3592847]
\end{abstract}

The discovery of extraordinary transmission through nanostructured metallic films by Ebbesen et al. ${ }^{1}$ in 1998 sparked a flurry of scientific activity as researchers tried to explain and utilize this phenomenon. The transmission from thin metallic films with subwavelength apertures has since been studied for a wide range of structures. ${ }^{2-11}$ Now, it is commonly accepted that the resonant behavior of these structures is mainly due to the presence of surface plasmon polaritons (SPPs) that are excited on the surface of the film. This knowledge has allowed researchers to design such structures to act as tunable filters, ${ }^{12}$ optical modulators, ${ }^{13}$ beam-splitters, ${ }^{14}$ or even form the basis of complex systems such as microscopes, ${ }^{15}$ photodetectors, ${ }^{16}$ and laser sources. ${ }^{17}$

A SPP is a propagating surface mode that results from the interactions between oscillating conduction electrons at a metal-dielectric interface and electromagnetic waves. Because, for a given frequency, the momentum of a SPP is greater than that of light, extra momentum must be added to the incident radiation if resonant excitation is desired. This can be achieved with a (quasi) periodic structure, such as a grating, which requires that the following condition be fulfilled: ${ }^{18}$

$$
k_{S P}=k_{\|} \pm m k_{G}^{(i)},
$$

where $k_{S P}$ is the SPP wave vector, $m$ is an integer, and $k_{G}^{(i)}$ is the reciprocal lattice vector of the structure; in this letter $i=p, q$, or $r$ related to the reciprocal lattice vector of periodic, quasiperiodic, or random slit arrangements, respectively. Consequently, there have been many studies of the transmission properties of such structures, including on single apertures surrounded by corrugations, ${ }^{3}$ one-dimensional (1D) periodic arrays of slits,, 19 and even quasiperiodic structures. ${ }^{4-8,10,11}$ Further, plasmonic structures are promising as elements for broadband couplers of light in applications, such as solar cells. ${ }^{20}$

The recent interest in quasiperiodic structures arises since the long-range order of these systems differs from the short range, unlike in periodic structures, which the order remains the same over different length scales. For a periodic structure with average distance $D, k_{G}^{(p)}=G=2 \pi / D$. In con-

${ }^{a)}$ Electronic mail: lefeber@amolf.nl. trast, quasiperiodic structures can have reciprocal lattice vectors $k_{G}^{(q)} \in(0, G)$ and $k_{G}^{(q)} \in(G, 2 G)$ that we relate to longrange and short-range order, respectively. Hence, if the geometry of the quasiperiodic structures is chosen correctly their resonant plasmonic behavior can strongly differ from that of the periodic arrays. Specifically it has been theorized that the increased complexity of these systems can result in a large array of reciprocal lattice vectors, leading to multiple plasmonic coupling resonances that span a broad range of wavelengths. $^{7}$

Recent experimental work in this field has been primarily focused on either extraordinary transmission ${ }^{4-6}$ or excitation of localized surface plasmons ${ }^{11}$ in two-dimensional quasiperiodic arrays of holes. Likewise, the studies of 1D quasiperiodic structures are either such that the sample geometry precludes plasmonic excitation ${ }^{10}$ or such that only the short-range order effects are observed. ${ }^{8}$

In this letter we study the effect of short- and long-range order on the zeroth order transmission spectra of 1D quasiperiodic, periodic, and randomized arrangements of subwavelength slits. We show that a careful design of the quasiperiodic slit distribution leads to broadband first order $[m=1$ in Eq. (1)] plasmonic excitation with these resonances spanning the visible and the near-infrared (NIR) regions. In addition, we identify plasmonic resonances that arise from the longrange order of our samples.

In Fig. 1 we show scanning electron microscopy (SEM) images of the slit arrangements, as suggested in Ref. 7, these

(a)
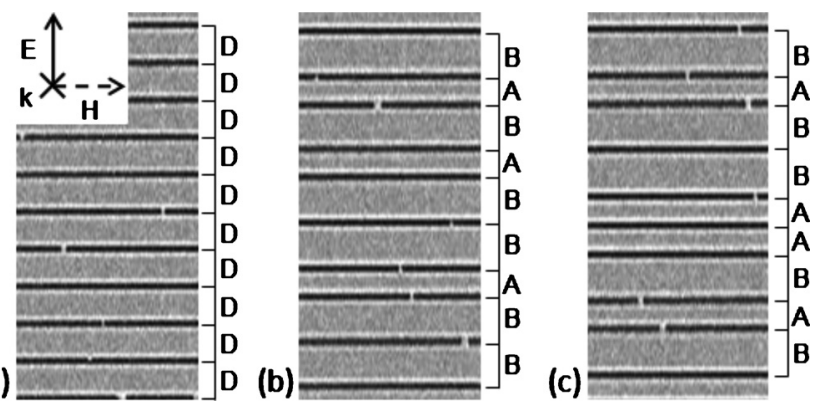

FIG. 1. SEM images of the (a) periodic, (b) Fibonacci, and (c) randomized slit arrangements. A, B, and D represent slit separation distances. The structures are illuminated by p-polarized light with the E-field lying in the plane of the slit arrangements, as shown in the inset. 
consist of 200 identical slits. To ensure sufficient transmission and stay in the subwavelength regime we use $120 \mathrm{~nm}$ wide, $1 \mu \mathrm{m}$ long slits in a $200 \mathrm{~nm}$ thick gold layer, which is positioned on top of a glass plate $(n=1.53)$. These samples were fabricated using focused ion beam techniques. Figures 1(a) and 1(c) show the periodic and random slit arrangement, respectively; in the former, all slits are a distance $D$ from their neighbors while in the latter slit separations vary randomly between $A$ and $B$, with a fixed ratio, $\phi$, such that the average separation remains $D$. Here, $\phi$ is the golden ratio $[=(1+\sqrt{5}) / 2]$ that is chosen since it allows us to relate measurements from these configurations with those for a quasiperiodic array of slits.

Although a quasiperiodic arrangement, such as the one we present in Fig. 1(b) appears disordered on short length scales, it really contains both short- and long-range order. For our quasiperiodic array we choose a Fibonacci sequence, in which distances $A$ and $B$, are arranged according to

$$
\left[S_{n}\right]=\left[S_{n-1}\right]\left[S_{n-2}\right] .
$$

Here $\left[S_{n}\right]$ is the $n$th sequence, and the first two terms in the series are $\left[S_{0}\right]=B$ and $\left[S_{1}\right]=A$. We use the same golden ratio as for the random structures, which allows us to express the average slit separation as

$$
D=\frac{\phi^{2}+1}{\phi+1} \times A .
$$

That is, by correctly setting $A$ we ensure that $D$ is the same for all three slit configurations. In what follows we use samples with $A=300,350,400$, and $450 \mathrm{~nm}$ (with $B=\phi \cdot A$ ), corresponding to $D=415,484,553$, and $621 \mathrm{~nm}$.

We focus normally incident p-polarized (see inset of Fig. 1) broadband (550-1700 nm) light onto the samples and spectrally resolve the transmission of the different arrays. In Figs. 2(a)-2(c) we show the spectra obtained from the periodic, Fibonacci and randomized slit configurations for different $D$. All configurations show dips for which a change in $D$ corresponds to a change in their central wavelength. These dips are attributed to the excitation of SPPs. ${ }^{8}$

To understand the positions of these features, we compute the structure factor by means of discrete Fourier transform of the slits positions, ${ }^{21}$

$$
S\left(k_{G}^{(i)}\right)=\sum_{a=1}^{n} e^{i k_{G}^{(i)} x_{a}}
$$

which allows us to identify the reciprocal lattice vectors of our slit arrays. Here $n$ and $x_{a}$ are the number of slits and their positions, respectively. In essence, by superimposing periodic arrays with different periods it is possible to design a structure with the same peaks in its discrete Fourier transform as a quasiperiodic structure. Indeed, the discrete Fourier transform tells us, which periodic arrays are superimposed. However, whereas the quasiperiodic structure can be constructed from identical slits with only two different slit-to-slit separations, a superposition of different periods would, in the case of only a few discrete periods, result in a very complicated structure. Further, the disorder in the quasiperiodic structures adds very small reciprocal lattice vectors over a broad range of frequencies to the structure factor in the region between the main peaks [Fig. 2(e)], in essence slightly broadening these peaks.

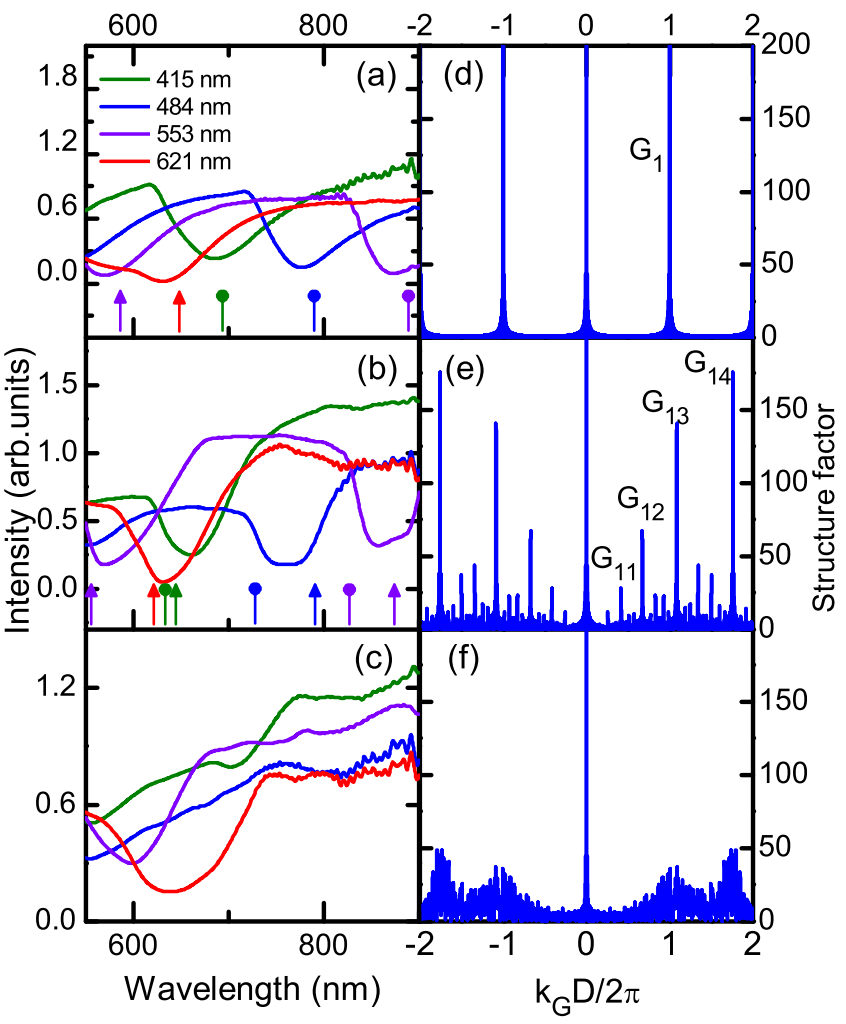

FIG. 2. (Color online) Transmission spectra obtained from the (a) periodic, (b) quasiperiodic, and (c) randomized slit arrays. We use normal (round) arrows to indicate the excitation wavelength of the SPP modes predicted using Eq. (1) on the air-gold (glass-gold) interface. Parts (d) to (f) show the structure factor for the structures corresponding to (a) to (c). The horizontal axis is plotted in units of $k_{G}^{(i)} / G$. In (e) $k_{1}^{(q)}, k_{2}^{(q)}, k_{3}^{(q)}$, and $k_{4}^{(q)}$ correspond to $k_{G}^{(q)} / G$ equal to $0.42,0.67,1.08$, and 1.67 , respectively.

In Figs. 2(d)-2(f), we show plots of the structure factor computed for arrays of 200 slits. As expected, the structure factor of the periodic grating [Fig. 2(d)] is narrowly peaked at $k_{G}^{(p)} / G=m$, where $m$ is an integer. Figure 2(e) shows a plot of the structure factor of the Fibonacci arrangement. Here we see clear peaks at the position $k_{3}^{(q)}$ and $k_{4}^{(q)}$, that are related to the short-range order of the array, and less pronounced peaks at $k_{1}^{(q)}$ and $k_{2}^{(q)}$, which arise due to long-range ordering. Although the randomized arrangement lacks the long-range order that is present in the Fibonacci arrangements, its structure factor [Fig. 2(f)] does contain peaks near $k_{3}^{(q)}$ and $k_{4}^{(q)}$. These peaks are expected since we use the same $A$ and $B$ as for the quasiperiodic arrangement, though they are broader since the distribution is randomized.

Using Eq. (1) (with $m=1$ ), we match the wave vectors obtained from the structure factor to the predicted excitation wavelength of SPPs on the metal-air and metal-glass interface, shown in Figs. 2(a)-2(c). In the simple case of the periodic grating [Fig. 2(a)] we find a good agreement between the predicted locations of the excitations of SPPs and the observed dips centers.

Although the resonant features in the transmission spectrum of the quasiperiodic and periodic slit arrays are similar, the additional reciprocal lattice vectors of the former structure must be accounted for correctly. First, the two air-gold coupling resonances (normal arrows) near 560 and $630 \mathrm{~nm}$ are related to $k_{3}^{(q)}$ while the other three resonances are due to $k_{2}^{(q)}$ and hence can be traced to the long-range order of this structure. Further, as is seen by the proximity of the differ- 


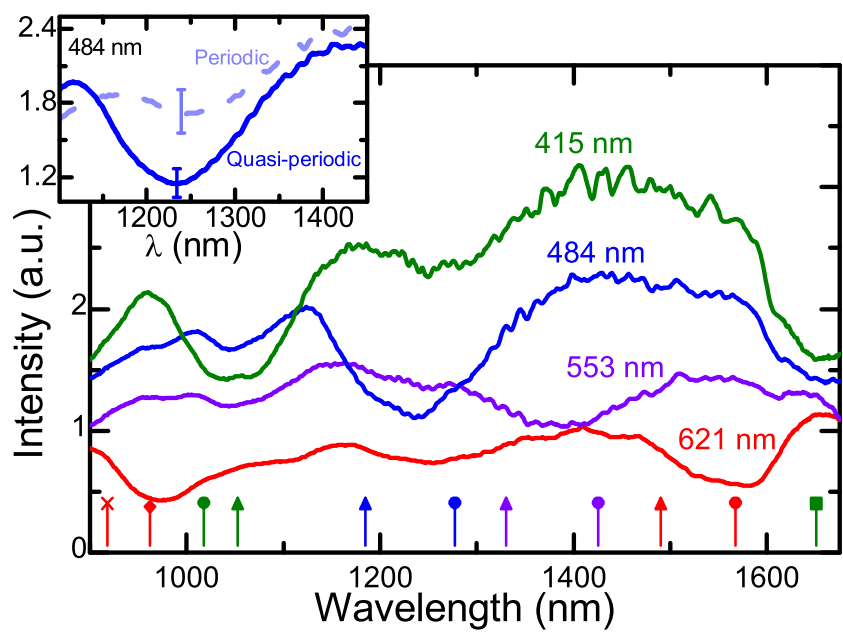

FIG. 3. (Color online) The transmission spectra from the Fibonacci arrangements for different average slit-to-slit distances. The arrows indicate the predicted wavelength for the excitation of a SPP. Normal (round) arrows correspond to a plasmonic coupling resonance at the glass-gold (air-gold) interface, via $k_{1}^{(q)}\left(k_{2}^{(q)}\right)$, the rightmost (square) arrow corresponds to a $k_{1}^{(q)}$ excitation at the glass-gold interface. At the left side, the cross (diamond) headed arrow corresponds to $k_{4}^{(q)}\left(k_{3}^{(q)}\right)$ on the air (glass) interface. In the inset we compare a typical resonance in the spectrum of the Fibonacci arrangement (solid line) and the spectrum of the grating (dashed line), both with $D=484 \mathrm{~nm}$.

ently headed arrows, we expect that each air-gold resonance will be located near a glass-gold resonance (round arrows) that arises from $k_{3}^{(q)}$. For example, the dip near $765 \mathrm{~nm}$ for the $D=484 \mathrm{~nm}$ curve is due to a resonance at the air-gold interface that is related to $k_{2}^{(q)}$ and one on the glass-gold interface related to $k_{3}^{(q)}$.

Lastly, the randomized array appears to show dips related to $k_{3}^{(q)}$ but, as expected, these features are broad compared to those in the other arrangements. We attribute this characteristic to the large width of the corresponding structure factor peak in Fig. 2(f) because the resonant coupling condition [Eq. (1)] is fulfilled for a large range of wavelengths.

To confirm the presence of resonances related to the long-range order of the Fibonacci arrangement, we measured the transmission spectra in the NIR, where the features related to $k_{3}^{(q)}$ and $k_{4}^{(q)}$ are absent. We show these results in the main part of Fig. 3 and indicate with arrows the location of the plasmonic resonances that are predicted to arise from $k_{1}^{(q)}$ and $k_{2}^{(q)}$. In the inset we compare one of these curves with similar data taken from a periodic array (dashed), and indeed see that, within errors, only data from the quasiperiodic structures shows resonant plasmonic features.

In conclusion, we have contrasted the transmission spectra from quasiperiodic arrays of slits with those from peri- odic and randomized arrangements. This comparison allowed us to identify plasmonic coupling resonances that arise due to the long-range order in the quasiperiodic structures. In turn, this allowed us to design quasiperiodic structures with first-order plasmonic coupling resonances that span both the visible and the NIR parts of the spectrum. We note that the properties of these structures can be further optimized by tuning the dimensions of the slits. ${ }^{19}$ Thus, these quasiperiodic arrays may be of use in the design of broadband plasmonic-based devices. In particular, the additional increase in reciprocal lattice vectors contained in this structures make them appealing for applications that require the absorption of light over a large spectral range, such as for solar-cells.

This work is part of the research program of the Stichting voor Fundamenteel Onderzoek der Materie (FOM), which is financially supported by the Nederlandse Organisatie voor Wetenschappelijk Onderzoek (NWO).

${ }^{1}$ T. W. Ebbesen, H. J. Lezec, H. F. Ghaemi, T. Thio, and P. A. Wolff, Nature (London) 391, 667 (1998).

${ }^{2}$ F. J. Garcia-Vidal, L. Martin-Moreno, T. W. Ebbesen, and L. Kuipers, Rev. Mod. Phys. 82, 729 (2010).

${ }^{3}$ H. J. Lezec, A. Degiron, E. Devaux, R. A. Linke, L. Martin-Moreno, F. J. García-Vidal, and T. W. Ebbesen, Science 297, 820 (2002).

${ }^{4}$ T. Matsui, A. Agrawal, A. Nahata, and Z. V. Vardeny, Nature (London) 446, 517 (2007).

${ }^{5}$ F. Przybilla, C. Genet, and T. W. Ebbesen, Appl. Phys. Lett. 89, 121115 (2006).

${ }^{6}$ N. Papasimakis, V. A. Fedotov, A. S. Schwanecke, N. I. Zheludev, and F. J. García de Abajo, Appl. Phys. Lett. 91, 081503 (2007).

${ }^{7}$ A. I. Fernández-Domínguez, I. Hernández-Carrasco, L. Martín-Moreno, and F. J. García-Vidal, Electromagnetics 28, 186 (2008).

${ }^{8}$ J. Li, S. Liu, C. Huang, T. Li, Q. Wang, and Y. Zhu, J. Opt. A, Pure Appl. Opt. 10, 075202 (2008).

${ }^{9}$ U. Schröter and D. Heitmann, Phys. Rev. B 58, 15419 (1998).

${ }^{10}$ C. Rockstuhl, F. Lederer, T. Zentgraf, and H. Giessen, Appl. Phys. Lett. 91, 151109 (2007).

${ }^{11}$ R. Dallapiccola, A. Gopinath, F. Stellacci, and L. Dal Negro, Opt. Express 16, 5544 (2008).

${ }^{12}$ E. Fu, J. Foley, and P. Yager, Rev. Sci. Instrum. 74, 3182 (2003).

${ }^{13}$ T. Nikolajsen, K. Leosson, and S. I. Bozhevolnyi, Appl. Phys. Lett. 85, 5833 (2004).

${ }^{14}$ Z. Sun, Appl. Phys. Lett. 89, 261119 (2006).

${ }^{15}$ B. Guo, Q. Gan, G. Song, J. Gao, and L. Chen, J. Lightwave Technol. 25, 830 (2007).

${ }^{16}$ T. Ishi, J. Fujikata, K. Makita, T. Baba, and K. Ohashi, Jpn. J. Appl. Phys., Part 2 44, L364 (2005)

${ }^{17}$ N. Yu, Q. J. Wang, M. A. Kats, J. A. Fan, S. P. Khanna, L. Li, A. G. Davies, E. H. Linfield, and F. Capasso, Nature Mater. 9, 730 (2010).

${ }^{18} \mathrm{H}$. Raether, Surface Plasmons on Smooth and Rough Surfaces and on Gratings (Springer, Berlin, 1988)

${ }^{19}$ Y. Pang, C. Genet, and T. W. Ebbesen, Opt. Commun. 280, 10 (2007).

${ }^{20}$ H. Atwater and A. Polman, Nature Mater. 9, 205 (2010).

${ }^{21}$ C. Kittel and P. McEuen, Introduction to Solid State Physics (Wiley, New York, 1986), Vol. 4. 


\section{Erratum: "Exploiting long-ranged order in quasiperiodic structures for broadband plasmonic excitation" [Appl. Phys. Lett. 98, 201108 (2011)]}

B. le Feber, ${ }^{\text {a) }}$ J. Cesario, H. Zeijlemaker, N. Rotenberg, and L. Kuipers Center for Nanophotonics, FOM Institute for Atomic and Molecular Physics (AMOLF), Science Park 104, 1098 XG Amsterdam, The Netherlands

(Received 15 June 2011; accepted 20 June 2011; published online 8 July 2011)

[doi:10.1063/1.3609319]

In Ref. 1, in the paragraph following Eq. (2), the first two terms of the Fibonacci sequence are incorrectly stated as $\left[S_{0}\right]=B$ and $\left[S_{1}\right]=A$. The correct first two terms are $\left[S_{0}\right]=A$ and $\left[S_{1}\right]=B$, for which Eq. (3) (and all subsequent equations) is correct as given in the original text. The analy- sis was performed using the correct terms, and therefore, the results and conclusions are unchanged.

${ }^{1}$ B. le Feber, J. Cesario, H. Zeijlemaker, N. Rotenberg, and L. Kuipers, Appl. Phys. Lett. 98, 201108 (2011).

${ }^{\text {a)} E l e c t r o n i c ~ m a i l: ~ l e f e b e r @ a m o l f . n l . ~}$ 